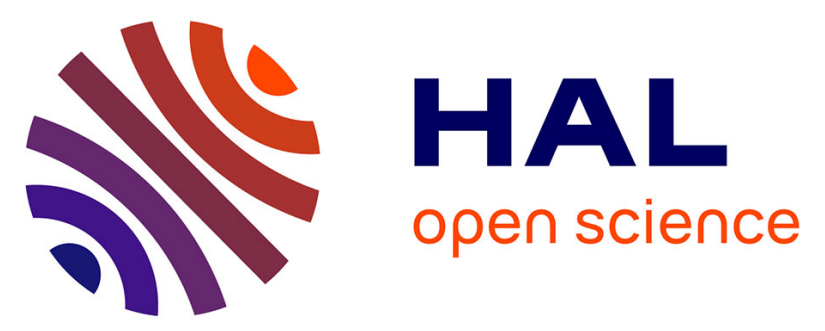

\title{
Passivity Analysis and Design of a Robust Nested Passivity-Based Controller for Trajectory Tracking of Autonomous Vehicles
}

Gilles Tagne, Reine Talj, Ali Charara

\section{- To cite this version:}

Gilles Tagne, Reine Talj, Ali Charara. Passivity Analysis and Design of a Robust Nested PassivityBased Controller for Trajectory Tracking of Autonomous Vehicles. 19th World Congress of the International Federation of Automatic Control (IFAC WC 2014), Aug 2014, Cape Town, South Africa. pp.9840-9846. hal-01061266

\section{HAL Id: hal-01061266 https://hal.science/hal-01061266}

Submitted on 16 Mar 2015

HAL is a multi-disciplinary open access archive for the deposit and dissemination of scientific research documents, whether they are published or not. The documents may come from teaching and research institutions in France or abroad, or from public or private research centers.
L'archive ouverte pluridisciplinaire HAL, est destinée au dépôt et à la diffusion de documents scientifiques de niveau recherche, publiés ou non, émanant des établissements d'enseignement et de recherche français ou étrangers, des laboratoires publics ou privés.

$$
\text { Copyright }
$$




\title{
Passivity Analysis and Design of a Robust Nested Passivity-Based Controller for Trajectory Tracking of Autonomous Vehicles
}

\author{
Gilles Tagne* Reine Talj* Ali Charara* \\ * Heudiasyc Laboratory, UMR CNRS 7253, Université de Technologie \\ de Compiègne, BP 20529, 60205 Compiègne, France (e-mail: \\ gilles.tagne@hds.utc.fr, reine.talj@hds.utc.fr, ali.charara@hds.utc.fr).
}

\begin{abstract}
This paper focuses on the lateral control of intelligent vehicles. The aim is to minimize the lateral displacement of the autonomous vehicle with respect to a given reference trajectory. The control input is the steering angle and the output is the lateral error displacement. After passivity analysis of the system to establish the properties of passivity between the inputs and outputs, we present design and validation of a robust lateral nested controller based on passivity, to ensure robust stability and good performances with respect to parametric variations and uncertainties encountered in driving applications. To validate the control strategy, the simulation of the closed-loop system on Matlab-Simulink has been made using the experimental data acquired on the vehicle DYNA of Heudiasyc laboratory (a Peugeot 308 ), according to several real driving scenarios. The validation shows robustness and good performances of the proposed control approach, and puts in evidence the improvement brought by the Nested PBC controller.
\end{abstract}

\section{INTRODUCTION}

The developments in the applications of ADAS (Advanced Driver Assistance Systems) and autonomous driving have been favored by technological advances in recent years. Three main steps are necessary to ensure an autonomous navigation: the perception and localization, the path planning and the vehicle control. The vehicle control can be divided into two tasks: longitudinal control and lateral control.

Lateral control consists on handling the vehicle using the steering wheel to follow the reference trajectory. In recent years, considerable researches have been made to provide lateral guidance of autonomous vehicles. In literature, many control strategies have been developed. Simple PID controller have been proposed in (Broggi et al. [1999]). We also have nested controller in (Marino et al. [2011]). Moreover, other classical techniques have been used. We can cite $H_{\infty}$ (Hima et al. [2011]), state feedback (Rajamani [2006]), Lyapunov stability based control (Benine-Neto et al. [2010]), fuzzy logic (Naranjo et al. [2008]), fuzzy Takagi-Sugeno LQ (Soualmi et al. [2012]), linear quadratic optimal predictive control (Kim et al. [2011]) and many others. Model Predictive Control (MPC) appears to be well suited to the trajectory following (Levinson et al. [2011]).

In (Hingwe and Tomizuka [1997], Tagne et al. [2013a]), Sliding Mode Control (SMC) has been applied to the

\footnotetext{
* This work was carried out in the framework of the Labex MS2T, which was funded by the French Government, through the program Investments for the future managed by the National Agency for Research (Reference ANR-11-IDEX-0004-02).
}

lateral control. This robust control strategy is well suited to driving applications, given its robustness against uncertainties and its capacity to reject disturbances. However, its main drawback is the chattering. Given the implicit resemblance between the SMC and the Immersion and Invariance $(I \& I)$ principle, in (Tagne et al. [2013b]), a controller based on the $I \& I$ approach have been developed to improve SMC perfomances.

Considering the high nonlinearity of the vehicle on one hand, and the uncertainties and disturbances in automotive applications on the other hand, an important issue to be considered in the control design is the robustness. The controller should be able to reject the disturbances and deal with parameter uncertainties and variations. For example, in (Levinson et al. [2011]), a recent presentation of Junior; Stanford's autonomous vehicle (the second at the DARPA Urban Challenge), is carried out for the purpose of ensuring robust autonomous driving.

Passivity is a concept that can be used in several areas of science and explains some physical phenomena. The theory of passivity is a framework for analyzing systems and designing controllers using a description of the inputsoutputs based on energy considerations. The main idea is that a very large number of physical systems have some properties of passivity between the inputs and outputs. We study the passivity of the system to analyze the frequency behavior, and determine the passive outputs (to easily control the system). The interest of passivity results from the feedback interconnection of passive systems. We can therefore seek the passivity of the system as a way to impose robust stability and good performances. This is particularly relevant in applications of lateral control of 
vehicles where the controller must ensure robust stability and good performances with respect to variations of speed, curvature, coefficient of friction of the road, wind and uncertainties on parameters.

The design of the $I \& I$ controller in (Tagne et al. [2013b]) allows to prove a very strong stability criterion of the closed-loop system. This result led us to study the properties of passivity of the different input-output maps of the system to design robust controllers. The main purpose of this paper is to establish the properties of passivity between the inputs and outputs. Finally, we develop a Passivity-Based Controller (PBC). To design the controller, we consider that the vehicle is equipped with sensors and/or observers to measure the yaw rate, the lateral error and its derivative. To validate the proposed approach, tests were made with real data acquired on the vehicle DYNA, on the tracks and circuits of CERAM ${ }^{1}$. The simulation results show the performance and robustness of the proposed approach.

This paper is organized as follows. Section 2 presents the dynamical models of the vehicle used for control design and the control problem definition. And then the passivity analysis is presented in Section 3. The control strategy is developed in Section 4. Section 5 presents the simulation results. Finally, we conclude in Section 6, with some remarks and future work directions.

\section{DYNAMIC MODEL OF VEHICLE AND CONTROL PROBLEM DEFINITION}

\subsection{Dynamic models of vehicle}

In this work, we use two vehicle models. To design the controller, a simple and widely used dynamic bicycle model from (Rajamani [2006]) is considered. This model is used to represent the lateral vehicle behavior and assumes that the vehicle is symmetrical, and tire's sideslip angles on the same axle are equal. The roll and pitch dynamics are neglected and angles are assumed to be small (steering, sideslip, yaw). With a linear tire force model we obtain a Linear Parameter Varying (LPV) model, where the longitudinal velocity $V_{x}$ is considered as a varying parameter. Dynamic equations in terms of slip angle and yaw rate of the bicycle model are given by:

$$
\begin{aligned}
& \dot{\beta}=-\frac{\mu\left(C_{f}+C_{r}\right)}{m V_{x}} \beta-\left(1+\frac{\mu\left(L_{f} C_{f}-L_{r} C_{r}\right)}{m V_{x}^{2}}\right) \dot{\psi}+\frac{\mu C_{f}}{m V_{x}} \delta \\
& \ddot{\psi}=-\frac{\mu\left(L_{f} C_{f}-L_{r} C_{r}\right)}{I_{z}} \beta-\frac{\mu\left(L_{f}^{2} C_{f}+L_{r}^{2} C_{r}\right)}{I_{z} V_{x}} \dot{\psi}+\frac{\mu L_{f} C_{f}}{I_{z}} \delta
\end{aligned}
$$

where $\beta, \psi$ and $\delta$ represent respectively the sideslip angle, the yaw angle of the vehicle and the steering wheel angle (control input). Table 1 presents vehicle parameters and nomenclature.

To validate the proposed controller in simulation, we used a more representative model, namely, the 4 -wheel model to represent the behavior of the vehicle and Dugoff's tire model for longitudinal and lateral forces.

\footnotetext{
1 CERAM -"Centre d'Essais et de Recherche Automobile de Mortefontaine" is an automobile testing and research center located in France.
}

Table 1. Vehicle Parameters and Nomenclature

\begin{tabular}{|c||c||c||c|}
\hline$V_{x}$ & Longitudinal velocity & - & {$[\mathrm{m} / \mathrm{s}]$} \\
\hline$\beta$ & Sideslip angle & - & {$[\mathrm{rad}]$} \\
\hline$\dot{\psi}$ & Yaw rate & - & {$[\mathrm{rad} / \mathrm{s}]$} \\
\hline$\delta$ & Steering wheel angle & - & {$[\mathrm{rad}]$} \\
\hline$\mu$ & Road friction coefficient & 1 & - \\
\hline $\mathrm{m}$ & Mass & 1719 & {$[\mathrm{~kg}]$} \\
\hline$I_{z}$ & Yaw moment of inertia & 3300 & {$\left[\mathrm{kgm}^{2}\right]$} \\
\hline$L_{f}$ & Front axle-COG distance & 1.195 & {$[\mathrm{~m}]$} \\
\hline$L_{r}$ & Rear axle-COG distance & 1.513 & {$[\mathrm{~m}]$} \\
\hline$C_{f}$ & Cornering stiffness of the front tire & 170550 & {$[\mathrm{~N} / \mathrm{rad}]$} \\
\hline$C_{r}$ & Cornering stiffness of the rear tire & 137844 & {$[\mathrm{~N} / \mathrm{rad}]$} \\
\hline
\end{tabular}

\subsection{Control problem definition}

The aim of the lateral control of intelligent vehicles is to minimize the lateral displacement of the autonomous vehicle with respect to a given reference trajectory. The lateral error dynamic at the center of gravity of the vehicle, with respect to a reference trajectory, is given by:

$$
\ddot{e}=a_{y}-a_{y_{\text {ref }}}
$$

where $a_{y}$ and $a_{y_{\text {ref }}}$ represent respectively the lateral acceleration of the vehicle, and the desired one on the reference trajectory. Assuming that the desired lateral acceleration of the vehicle can be written as $a_{y_{r e f}}=V_{x}^{2} \rho$, where $\rho$ is the curvature of the road, and given that $a_{y}=V_{x}(\dot{\beta}+\dot{\psi})$, we have:

$$
\ddot{e}=V_{x}(\dot{\beta}+\dot{\psi})-V_{x}^{2} \rho
$$

Replacing $\dot{\beta}$ by its expression in equation (1), the new system state variables become $x=(\beta, \dot{\psi}, \dot{e}, e)^{\top}$, corresponding to the sideslip angle, the yaw rate, the lateral error and its derivative. The new system has the following dynamics:

$$
\dot{x}=A x+B_{1} \delta+B_{2} \rho
$$

where,

$A=\left[\begin{array}{cccc}-\frac{\mu\left(C_{f}+C_{r}\right)}{m V_{x}} & -1-\frac{\mu\left(L_{f} C_{f}-L_{r} C_{r}\right)}{m V_{x}^{2}} & 0 & 0 \\ -\frac{\mu\left(L_{f} C_{f}-L_{r} C_{r}\right)}{I_{z}} & -\frac{\mu\left(L_{f}^{2} C_{f}+L_{r}^{2} C_{r}\right)}{I_{z} V_{x}} & 0 & 0 \\ -\frac{\mu\left(C_{f}+C_{r}\right)}{m} & -\frac{\mu\left(L_{f} C_{f}-L_{r} C_{r}\right)}{m V_{x}} & 0 & 0 \\ 0 & 0 & 1 & 0\end{array}\right]$

$\mathrm{B}_{1}=\left[\begin{array}{c}\frac{\mu C_{f}}{m V_{x_{f}}} \\ \frac{\mu L_{f} C_{f}}{I_{z_{f}}} \\ \frac{\mu C_{f}}{m} \\ 0\end{array}\right], \quad B_{2}=\left[\begin{array}{c}0 \\ 0 \\ -V_{x}^{2} \\ 0\end{array}\right]$

The aim of the lateral control is to cancel the lateral error displacement. Then, for a given curvature $\rho$ and longitudinal velocity $V_{x}$, the desired behavior corresponds to $\dot{e}_{1}=e_{1}=0$. Hence, it is easy to prove that the desired equilibrium point is:

$$
(\beta, \dot{\psi}, \dot{e}, e)^{\top}=\left(\beta^{\star}, \dot{\psi}^{\star}, 0,0\right)^{\top}
$$

with 


$$
\beta^{\star}=\left(L_{r}-\frac{L_{f} m V_{x}^{2}}{\mu C_{r}\left(L_{f}+L_{r}\right)}\right) \rho
$$

At the equilibrium point, the control input is:

$$
\delta^{\star}=\frac{L_{f} C_{f}-L_{r} C_{r}}{L_{f} C_{f}} \beta^{\star}+\frac{L_{f}^{2} C_{f}+L_{r}^{2} C_{r}}{L_{f} C_{f} V_{x}} \dot{\psi}^{\star}
$$

Hence, define the new error variables:

$$
\left\{\begin{array}{l}
\tilde{\beta}=\beta-\beta^{\star} \\
\dot{\tilde{\psi}}=\dot{\psi}-\dot{\psi}^{\star} \\
\tilde{\delta}=\delta-\delta^{\star}
\end{array}\right.
$$

The error dynamics of the system (4) having the origin as equilibrium point $(\tilde{\beta}, \dot{\tilde{\psi}}, \dot{e}, e)^{\top}=(0,0,0,0)^{\top}$ become:

$$
\dot{\tilde{x}}=A \tilde{x}+B_{1} \tilde{\delta}
$$

where, $A$ and $B_{1}$ have been defined above (4).

\section{PASSIVITY ANALYSIS}

Theory of passivity is a framework for analyzing physical systems and designing controllers using a description of the input-output relationship based on energy considerations. The Kalman-Yakubovich-Popov (KYP) lemma is considered to be one of the pillars for control and systems theory. To demonstrate the passivity of an output, this lemma is considered. It establishes an equivalence between the conditions in the frequency domain (positive realness of a system), in the time domain (an input-output relationship of the system), and in the state-space representation where conditions on the matrices describing the system should be verified. The Lemma is given as follows:

Lemma 1. (KYP lemma) Lozano et al. [2000]

\section{In the frequency domain}

Consider the transfer function $\mathrm{H}(\mathrm{s})$ and the operator returning the real part $\Re()$. H(s) is Positive Real (PR) if and only if:

$$
\left\{\begin{array}{l}
H(s) \text { is stable } \\
\Re[H(j \omega)] \geq 0, \forall \omega \in R
\end{array}\right.
$$

\section{In the time domain}

A system with input $u$ and output $y$ where $u(t), y(t) \in R^{n}$ is passive if there is a constant $v$ such that

$$
\int_{0}^{T} y^{T}(t) u(t) \geq v
$$

for all function $u$ and all $T \geq 0$.

\section{In the state-space representation}

Let us consider a system described by the following statespace representation: $\dot{x}=A x+B u$ and $y=C x+D u$. Where $x \in R^{n}, u, y \in R^{m}$ with $n \geq m$. The transfer function $H(s)=C^{T}(s I-A)^{-1} B+D$, with $A \in R^{n \times n}, B \in$ $R^{n \times m}, C \in R^{m \times n}, D \in R^{m \times m}$ is PR with $H(s) \in R^{m \times m}$, if and only if there exists matrices $P>0, P \in R^{n \times n}$, $L \in R^{n \times m}$ and $W \in R^{m \times m}$ such that:

$$
\left\{\begin{array}{l}
P A+A^{T} P=-L L^{T} \\
P B-C^{T}=-L W \\
D+D^{T}=W^{T} W
\end{array}\right.
$$

Remark : In view of the above conditions, it is clear that unstable systems or non-minimum phase systems are not positive real.

\subsection{Strong Strict Passivity of the map $\tilde{\delta} \rightarrow \ddot{e}$}

\section{Proposition 1. The map $\tilde{\delta} \rightarrow \ddot{e}$ is Strongly Strictly Passive.}

The proof of strong strict passivity is established showing that the transfer function $H_{0}(s)$ of the map $\tilde{\delta} \rightarrow \ddot{e}$ is Strongly Strictly Positive Real (SSPR).

Assume that the road coefficient of friction $\mu=1$. Combining dynamic equations of the bicycle model in terms of slip angle and yaw rate (1) with the equation of lateral error dynamic (3), and after some calculations, one can find that the transfer function $H_{0}(s)$ between $\tilde{\delta}$ as input and $\ddot{e}$ as output is given by:

$$
H_{0}(s)=\frac{\ddot{e}(s)}{\tilde{\delta}(s)}=\frac{a s^{2}+b s+c}{s^{2}+d s+f}
$$

where,

$$
\left\{\begin{array}{l}
a=\frac{C_{f}}{m} \\
b=\frac{L_{r} C_{f} C_{r}\left(L_{f}+L_{r}\right)}{m I_{z} V_{x}} \\
c=\frac{C_{f} C_{r}\left(L_{f}+L_{r}\right)}{m I_{z}} \\
d=\frac{\left(C_{f}+C_{r}\right)}{m V_{x}}+\frac{\left(L_{f}^{2} C_{f}+L_{r}^{2} C_{r}\right)}{I_{z} V_{x}} \\
f=\frac{C_{f} C_{r}\left(L_{f}+L_{r}\right)^{2}}{m I_{z} V_{x}^{2}}+\frac{\left(L_{r} C_{r}-L_{f} C_{f}\right)}{I_{z}}
\end{array}\right.
$$

The transfer function $H_{0}(s)$ has a relative degree equal to 0 . Consequently, according to the KYP Lemma applied in the frequency domain, the proof of SSPR is tantamount to verifying :

$$
\Re\left[H_{0}(j \omega)\right] \geq \zeta>0, \forall \omega \in(-\infty,+\infty),
$$

Whatever the uncertainties and variations encountered, the model parameters $\left(C_{f}, C_{r}, L_{f}, L_{r}, m, I_{z}\right)$ are always positive. Thus, the coefficients $a, b, c, d, f$ of $H_{0}(s)$ are always positive. Note that $f$ is always positive even if the second fraction of $f$ could become negative. According to the criterion of Routh-Hurwitz, zeros and poles of $H_{0}(s)$ are strictly stable, so $H_{0}(s)$ is minimum phase and Hurwitz. Setting $s=j \omega$, the frequency response function becomes

$$
H_{0}(j \omega)=\frac{-a \omega^{2}+b j \omega+c}{-\omega^{2}+d j \omega+f}=\frac{R_{N 0}+j I_{N 0}}{R_{D}+j I_{D}}
$$

where $R_{N 0}, I_{N 0}, R_{D}$ and $I_{D}$ are the real and imaginary parts of the numerator and the denominator respectively, given by: 


$$
\begin{gathered}
R_{N 0}=c-a \omega^{2}, I_{N 0}=b \omega \\
R_{D}=f-\omega^{2}, I_{D}=d \omega
\end{gathered}
$$

Hence,

$$
\Re\left[H_{0}(j \omega)\right]=\frac{R_{N 0} R_{D}+I_{N 0} I_{D}}{R_{D}^{2}+I_{D}^{2}}
$$

Knowing that $R_{D}^{2}+I_{D}^{2}>0, \Re\left[H_{0}(j \omega)\right]$ is positive if and only if $R_{N 0} R_{D}+I_{N 0} I_{D}>0$.

$$
R_{N 0} R_{D}+I_{N 0} I_{D}=a \omega^{4}+(b d-a f-c) \omega^{2}+c f
$$

Given the order of magnitude of the parameters mentioned above and whatever is $V_{x}>0$, one can find that:

$$
\left\{\begin{array}{l}
a>0 \\
c f>0 \\
b d-a f-c \text { is not always positive. }
\end{array}\right.
$$

Given that $(b d-a f-c)$ is not always positive, let $x=\omega^{2}$, hence

$R_{N 0} R_{D}+I_{N 0} I_{D}=f(x)=a x^{2}+(b d-a f-c) x+c f(19)$

The derivative of this function is zero for

$$
x_{0}=\frac{b d-a f-c}{-2 a}
$$

So, the unique extremum of the function is

$$
f\left(x_{0}\right)=\frac{(b d-a f-c)^{2}}{-4 a}+c f>0
$$

Moreover, given that $f(0)=c f>0$ and $f(\infty) \rightarrow a>0$, then

$$
f(x)=R_{N 0} R_{D}+I_{N 0} I_{D} \geq \zeta_{0}>0
$$

Given that

$$
\lim _{\omega \rightarrow \infty} \Re\left[H_{0}(j \omega)\right]=a>0
$$

and

$$
\lim _{\omega \rightarrow 0} \Re\left[H_{0}(j \omega)\right]=\frac{c}{f}>0
$$

one can prove that:

$$
\Re\left[H_{0}(j \omega)\right] \geq \zeta>0, \forall \omega \in(-\infty,+\infty)
$$

hence, the transfer function $H_{0}(s)$ is Strongly Strictly Positive Real (SSPR), yielding to the desired result.

\subsection{Passivity of the map $\tilde{\delta} \rightarrow \dot{e}$}

Proposition 2. The map output $\tilde{\delta} \rightarrow \dot{e}$ is passive (P).

The transfer function $H_{1}(s)$ of the output $\dot{e}$ with respect to the input $\tilde{\delta}$ is given by:

$$
H_{1}(s)=\frac{1}{s} H_{0}(s)
$$

The transfer function $H_{1}(s)$ is a cascade connection of an integrator with the strongly strictly positive real transfer function $H_{0}(s)$. Hence, $H_{1}(s)$ is positive real, yielding the passivity of the map $\tilde{\delta} \rightarrow \dot{e}$, the desired result. For more details see Lozano et al. [2000].
3.3 Strict Passivity of the map $\tilde{\delta} \rightarrow \dot{\tilde{\psi}}$

Proposition 3. The map $\tilde{\delta} \rightarrow \dot{\tilde{\psi}}$ is Strictly Passive (SP).

Proof.

The transfer function $H_{2}(s)$ of the yaw rate error $\dot{\tilde{\psi}}$ relative to the input $\tilde{\delta}$ is given by:

$$
H_{2}(s)=\frac{\dot{\tilde{\psi}}(s)}{\tilde{\delta}(s)}=\frac{g s+h}{s^{2}+d s+f}
$$

where,

$$
\left\{\begin{array}{l}
d \text { and } f \text { defined in }(13), \\
g=\frac{L_{f} C_{f}}{I_{z}} \\
h=\frac{\left(C_{f}+C_{r}\right)\left(L_{f}+L_{r}\right)}{m I_{z} V_{x}}
\end{array}\right.
$$

The coefficients $d, f, g$ and $h$ are always positive. Then, according to the Routh-Hurwitz criterion, poles of $\mathrm{H}_{2}(s)$ are stable. The proof of Strict Passivity of the map $\tilde{\delta} \rightarrow \tilde{\tilde{\psi}}$ is equivalent to the proof of Strict Positive Realness (SPR) of the transfer function $H_{2}(s)$. Given that the transfer function $H_{2}(s)$ has a relative degree equal to 1 , the SPR is established verifying the following:

(P1) $\Re\left[H_{2}(j \omega)\right]>0, \forall \omega \in(-\infty,+\infty)$,

(P2) $\lim _{\omega \rightarrow \infty} \omega^{2} \Re\left[H_{2}(j \omega)\right]>0$.

Indeed, the frequency response function of $H_{2}(s)$ for $s=$ $j \omega$ is

$$
H_{2}(j \omega)=\frac{g j \omega+h}{-\omega^{2}+d j \omega+f}=\frac{R_{N 2}+j I_{N 2}}{R_{D}+j I_{D}}
$$

where $R_{N 2}, I_{N 2}, R_{D}$ and $I_{D}$ are the real and imaginary parts of the numerator and the denominator respectively, given by:

$$
\begin{array}{r}
R_{N 2}=h, I_{N 2}=g \omega \\
R_{D}=f-\omega^{2}, I_{D}=d \omega
\end{array}
$$

Hence,

$$
\Re\left[H_{2}(j \omega)\right]=\frac{R_{N 2} R_{D}+I_{N 2} I_{D}}{R_{D}^{2}+I_{D}^{2}}
$$

As above, $R_{D}^{2}+I_{D}^{2}>0$, then $\Re\left[H_{2}(j \omega)\right]$ is positive if and only if $R_{N 2} R_{D}+I_{N 2} I_{D}>0$.

$$
R_{N 2} R_{D}+I_{N 2} I_{D}=(g d-h) \omega^{2}+f h
$$

Given the order of magnitude of parameters mentioned above and whatever is $V_{x}>0$, one can find that:

$$
\left.\begin{array}{c}
g d-h>0 \\
f h>0
\end{array}\right\} \Rightarrow \Re\left[H_{2}(j \omega)\right]>0, \forall \omega \in(-\infty,+\infty),
$$

verifying (P1). Furthermore,

$$
\lim _{\omega \rightarrow \infty} \omega^{2} \Re\left[H_{2}(j \omega)\right]=g d-h>0,
$$

verifying $(\mathrm{P} 2)$. Hence, $H_{2}(s)$ is a Strictly Positive Real transfer function. 
Note that $\lim _{\omega \rightarrow \infty} \Re\left[H_{2}(j \omega)\right]=0$. Then, the Strict Positive Realness (SPR) of $H_{2}(s)$ is not Strong, yielding the desired result of Strict Passivity of the $\operatorname{map} \tilde{\delta} \rightarrow \dot{\tilde{\psi}}$.

\subsection{Characteristics of the sideslip angle error $\tilde{\beta}$,}

Proposition 4. The map $\tilde{\delta} \rightarrow \tilde{\beta}$ is not passive.

Proof. The transfer function $H_{3}(s)$ of $\tilde{\beta}$, relative to the input $\tilde{\delta}$ is given by:

$$
H_{3}(s)=\frac{\tilde{\beta}(s)}{\tilde{\delta}(s)}=\frac{k s+l}{s^{2}+d s+f}
$$

where,

$$
\left\{\begin{array}{l}
d \text { and } f \text { defined in (13), } \\
k=\frac{C_{f}}{m V_{x}} \\
l=\frac{L_{r} C_{f} C_{r}\left(L_{f}+L_{r}\right)}{m I_{z} V_{x}^{2}}-\frac{L_{f} C_{f}}{I_{z}}
\end{array}\right.
$$

The transfer function $H_{3}(s)$ has a relative degree equal to 1. The variables $d, f$ and $k$ are always positive, but $l$ can be either positive or negative depending on the value of $V_{x}$ and other parameters. When $l<0, H_{3}(s)$ has a negative zero, and is not positive real, what proves the proposition.

The zero of the transfer function $H_{3}(s)$ is stable if and only if $l$ is positive, i.e.

hence,

$$
-\frac{L_{f} C_{f}}{I_{z}}+\frac{L_{r} C_{f} C_{r}\left(L_{f}+L_{r}\right)}{m I_{z} V_{x}^{2}}>0
$$

$$
V_{x}<\sqrt{\frac{L_{r} C_{r}\left(L_{f}+L_{r}\right)}{L_{f} m}} .
$$

This fact can be understood as if the map $\tilde{\delta} \rightarrow \tilde{\beta}$ is passive when the speed is limited as in (34), and this characteristic is stolen at high speeds. Using the parameters given in Table I, the speed limit of equation (34) corresponds to $V_{x}<16.5 \mathrm{~m} / \mathrm{s} \simeq 60 \mathrm{~km} / \mathrm{h}$.

\section{Proposition 5. The map $\dot{\tilde{\psi}} \rightarrow \ddot{e}$ is Strictly Passive and the map $\dot{\tilde{\psi}} \rightarrow \dot{e}$ is Passive.}

Proof. The proof is established at the same manner of the previous subsections.

To summarize, considering the error system (8), the passivity maps can be resumed in the Fig. 1.

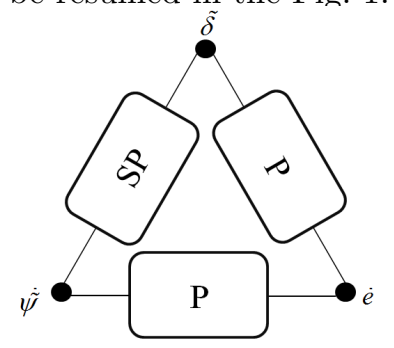

Fig. 1. Passivity maps of the system

\section{CONTROL STRATEGY}

Probably the main property used in PBC for passive systems is the fact that the feedback interconnection of passive systems is passive. So passivity is invariant under negative feedback interconnection. Therefore, passive systems can be decomposed into passive subsystems. Thus, in this methodology, the controller can be designed as a passive system. However, it will be also useful to know that interconnections not only preserve the passivity properties of the subsystems but, in certain cases, passivity can be strengthened.

Considering the Fig. 1 and knowing that the yaw rate dynamic is faster than the lateral error dynamic, we can decompose the lateral controller into two passive nested controllers. This is a major interest since $e$ and $\dot{\tilde{\psi}}$ are controlled simultaneously. We can therefore create two separate controllers for each own dynamic based on its characteristic of passivity. In this subsection, we develop a control strategy of an autonomous vehicle using two passive controllers to ensure guidance and stability (lateral error and yaw rate error) in two separate loops. The outer controller minimizes lateral error. The inner controller minimizes the yaw rate error by providing the corresponding steering angle. The particularity of such a strategy is doubled: it helps to design a robust controller while preserving the passivity properties of the closedloop system to ensure good performance. If necessary, two controllers of different natures can be used, in order to benefit from the robustness of each. It also allows an independent control of the lateral error and yaw rate error. Consequently, the scheme in Fig. 2 illustrates the proposed control strategy.

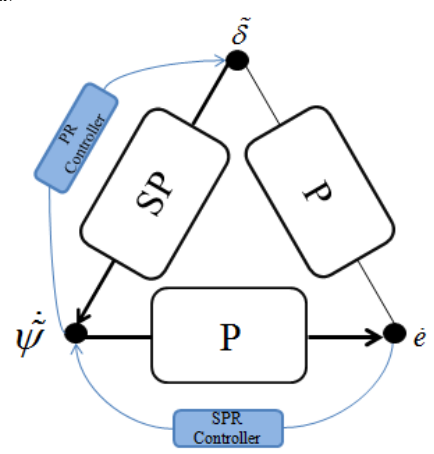

Fig. 2. Nested PBC control strategy

Proposition 6. Consider the diagram in Fig. 2, where the control is achieved by two PI controllers, the closed-loop system is stable and passive.

The proof is relatively simple, the corollary 4.1 in (Lozano et al. [2000]) allows to show it.

\section{Synthesis of the outer controller:}

This controller is designed to cancel the lateral error $e$. The control input is the yaw rate error.

Subsystem $\sum_{1}: \dot{\tilde{\psi}} \longmapsto \dot{e}$ is passive $(\mathrm{P})$, so any Input Strictly Passive (ISP) controller guarantees stability and passivity of the closed-loop system. In this paper, we choose a simple PI. We remind that a PI applied to the passive output $\dot{e}$ is equivalent to a $\mathrm{PD}$ applied to the 
output $e$. So, the control input given by the outer controller is:

$$
\dot{\tilde{\psi}}=-K_{D 1} \dot{e}-K_{P 1} e
$$

where $K_{D 1}$ and $K_{P 1}$ are positive gains.

\section{Synthesis of the inner controller:}

This controller is designed to cancel yaw rate error with respect to the reference given by the outer controller. The control input is the steering angle $\tilde{\delta}$.

Subsystem $\sum_{2}: \tilde{\delta} \longmapsto \dot{\tilde{\psi}}$ is strictly passive (SP), so any passive $(\mathrm{P})$ controller guarantees stability and passivity of the closed-loop system. In this paper, we choose a PI. The control input given by the inner controller is:

$$
\tilde{\delta}=-K_{I 2} \int \dot{\tilde{\psi}}-K_{P 2} \dot{\tilde{\psi}}
$$

where $K_{I 2}$ and $K_{P 2}$ are positive gains.

Given the strict passivity (SP) of the output $\dot{\tilde{\psi}}$ for an input $\tilde{\delta}$, the closed-loop system with a PI controller is stable and passive. Note that any passive controller, a simple proportional for example, would achieve the same result. The addition of integral action, well known to reject constant disturbances, also has the advantage that the controller can be implemented without the knowledge of $\delta^{\star}$ which depends on the uncertain model parameters.

\section{SIMULATION RESULTS}

To validate our control law, the experimental data used were acquired by the vehicle DYNA (a Peugeat 308) on the CERAM test circuits. This vehicle is equipped with different sensors and observers providing all the dynamic variables of the vehicle and its traveled path. The simulations in closed-loop are performed using as a reference, the real data with the full vehicle model. For the control law, we used the following values of the gains: $K_{D 1}=0.08, K_{P 1}=10, K_{P 2}=5$ and $K_{I 2}=1$. To highlight the improvements brought by the PBC controller, a comparison with a previously developed Immersion and Invariance $(I \& I)$ controller was made (Tagne et al. [2013b]).

\subsection{Test of the controller with nominal parameters}

This test (Fig. 3) was made with the purpose of testing the ability of the controller to track the reference trajectory during normal driving with known nominal parameters. Note that in this scenario we have a large turn to test the ability of the controllers to ensure proper path tracking for hard maneuvers. Fig. 3-(a) shows the longitudinal speed variations. Fig. 3-(b) presents different curves: the reference path and the trajectories followed by the controlled vehicle with two different controllers; the lateral error and the yaw angle error. Both controllers ensure the reference tracking with low errors (the lateral displacement of the controlled vehicle remains less than $10 \mathrm{~cm}$ in this test conditions).

In this scenario, the assumption of small angles is not respected (the steering angle is greater than 12 degrees (a)

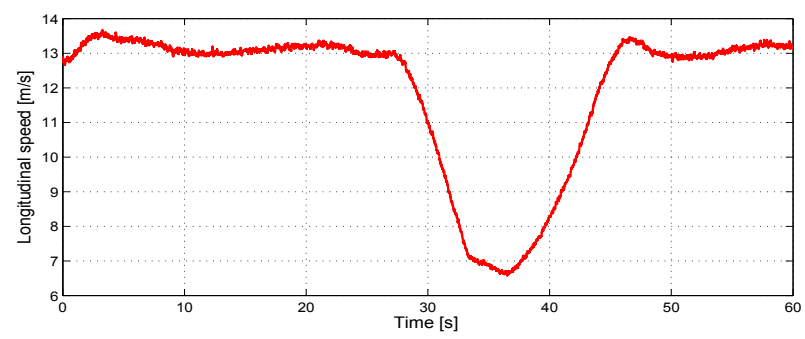

(b)
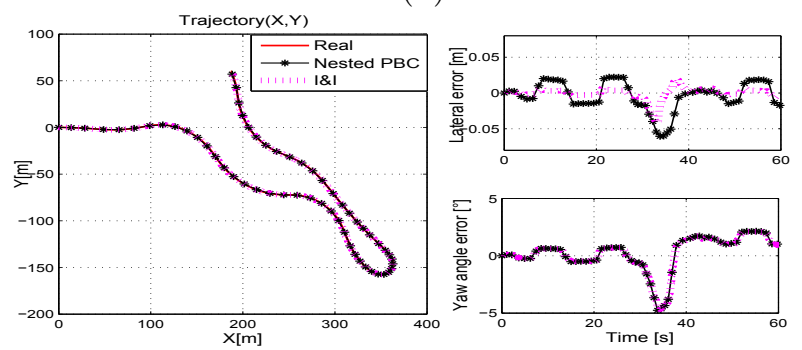

Fig. 3. (a) Longitudinal speed, (b) Trajectories ; reference and closed-loop simulations

during the turning). In spite of that, the controllers are able to follow the path with low errors. Both nonlinear controllers are robust to variations in longitudinal speed. So, this test show the good performance of the $I \& I$ and Nested PBC controller during normal driving and at varying speeds for known nominal parameters. We will evaluate the robustness of these controllers with respect to parametric uncertainties.

\subsection{Robustness to vehicle parameter uncertainties}

As mentioned previously, one of the major challenges for trajectory tracking is the robustness of the controller. In this subsection, we evaluate the robustness of the controllers with respect to parametric uncertainties of the vehicle. It is important to note that the parametric uncertainties can be due to the fact that the parameters may vary, but are considered to be fixed for the command. This is the case of the mass of the vehicle for example.

It is difficult to estimate accurately the cornering stiffness of the tires. Moreover, this parameter varies greatly depending on the type of the road, the vertical load, camber, etc. It is therefore important to assess the robustness of the controllers over cornering stiffness and mass variations. Fig. 4 presents lateral errors with uncertainties of $+/-10 \%$ on the value of the cornering stiffness. For uncertainties in the order of $10 \%$, on the value of cornering stiffness or mass, Nested PBC controller is able to follow the path with similar errors (to those of nominal conditions). The errors of the $I \& I$ remain acceptable.

Several other tests were performed, including scenarios of driving at high speed as well as situations where there is a saturation of lateral forces. With the $I \& I$ controller, the error depends on the value of the parametric uncertainty. Therefore with sensitivity study, we can estimate in advance the maximum error according to the knowledge of the parametric uncertainty. The passive controller (Nested PBC) is more robust to parameter uncertainties. Indeed, the inputs of the controller depend only on passive outputs 


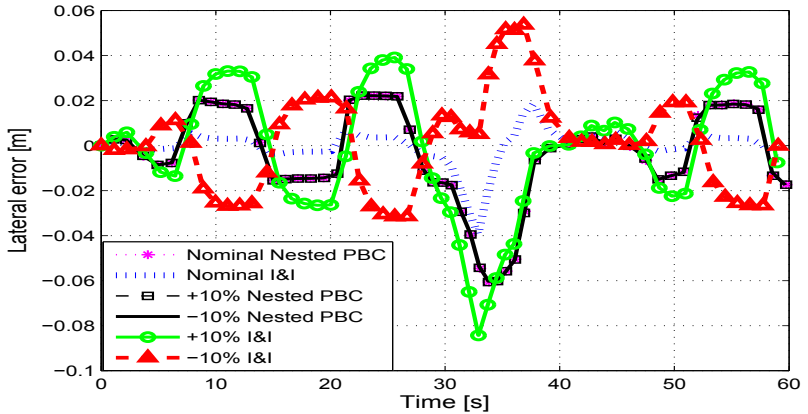

Fig. 4. Robustness against uncertainties of cornering stiffness $(+/-10 \%)$

(errors), which do not depend on uncertain parameters. However, the input of the $I \& I$ controller depends on these parameters. Furthermore, the PBC controller also has the advantage of using less measurements than the previous one. Indeed, with the $I \& I$ controller, the estimation of the sideslip angle is necessary. So, these results put in evidence the improvement brought by the Nested PBC controller.

\section{CONCLUSION}

In this paper, after a detailed study of the passivity properties of the model, a controller based on these properties has been proposed to ensure robust trajectory following of autonomous vehicles. The design of the controller has been presented. It guarantees robust stability and the passivity of the closed-loop system.

Results have shown that a greater consideration of structural features of the model during the design of the controller, and the passivity properties of the closed-loop system, can significantly improve the robustness of the controller for autonomous driving application. To illustrate this improvement, a comparison has been made with the $I \& I$ controller developed previously. The validation has shown robustness and good performance of the proposed Nested PBC controller.

The study of passivity is done considering the vehicle speed as a varying parameter. This is a non-restrictive assumption because the lateral dynamic is relatively faster than longitudinal dynamic. In this paper, the study of passivity properties has been done considering the road coefficient of friction $\mu=1$. An analysis for variant $\mu$ will be studied by the authors. In addition, a study of robustness based on sensitivity analysis will be performed. We will also evaluate the robustness against sensor noise like in (Althoff and Dolan [2011]). Very soon, we will test these control laws on the laboratory robotized vehicle.

\section{REFERENCES}

Matthias Althoff and John M. Dolan. Set-based computation of vehicle behaviors for the online verification of autonomous vehicles. In Int. IEEE Conference on Intelligent Transportation Systems (ITSC), pages 11621167, 2011.

Andre Benine-Neto, Stefano Scalzi, Said Mammar, and Mariana Netto. Dynamic controller for lane keeping and obstacle avoidance assistance system. In Int. IEEE Conference on Intelligent Vehicles Symposium (IV), pages 1363-1368, 2010.
Alberto Broggi, Massimo Bertozzi, and Alessandra Fascioli. The ARGO autonomous vehicle's vision and control systems. Int. Journal of Intelligent Control and Systems, 3(4):409-441, 1999.

Salim Hima, Benoit Lusseti, Benoit Vanholme, Sebastien Glaser, and Said Mammar. Trajectory Tracking for Highly Automated Passenger Vehicles. In International Federation of Automatic Control (IFAC) World Congress, pages 12958-12963, Milano, 2011.

Pushkar Hingwe and M. Tomizuka. Experimental evaluation of a chatter free sliding mode control for lateral control in AHS. In American Control Conference (ACC), 1997.

Dongwook Kim, Juyoung Kang, and Kyoungsu Yi. Control strategy for high-speed autonomous driving in structured road. In Int. IEEE Conference on Intelligent Transportation Systems (ITSC), 2011.

Jesse Levinson, Jake Askeland, Jan Becker, Jennifer Dolson, David Held, Soeren Kammel, J Zico Kolter, Dirk Langer, Oliver Pink, Vaughan Pratt, Michael Sokolsky, Ganymed Stanek, David Stavens, Alex Teichman, Moritz Werling, and Sebastian Thrun. Towards Fully Autonomous Driving : Systems and Algorithms. In Int. IEEE Conference on Intelligent Vehicles Symposium (IV), pages 163-168, Baden-Baden, 2011.

Rogelio Lozano, Bernard Brogliato, Olav Egeland, and Bernhard Maschke. Dissipative Systems Analysis and Control: Theory and Applications. Springer, 2000.

Riccardo Marino, Stefano Scalzi, and Mariana Netto. Nested PID steering control for lane keeping in autonomous vehicles. Control Engineering Practice, 19 (12):1459-1467, December 2011. ISSN 09670661.

J.E. Naranjo, C. Gonzalez, R. Garcia, and T. de Pedro. Lane-Change Fuzzy Control in Autonomous Vehicles for the Overtaking Maneuver. Iint. IEEE Transactions on Intelligent Transportation Systems, 9(3):438450, September 2008. ISSN 1524-9050.

R Rajamani. Vehicle dynamics and control. Springer, 2006. ISBN 978-0-387-26396-0.

B. Soualmi, C. Sentouh, J. C. Popieul, and S. Debernard. Fuzzy Takagi-Sugeno LQ controller for lateral control assistance of a vehicle. In Int. IEEE Conference on Intelligent Vehicles Symposium (IV), pages 377-382, Alcalá de Henares, 2012.

Gilles Tagne, Reine Talj, and Ali Charara. Higher-Order Sliding Mode Control for Lateral Dynamics of Autonomous Vehicles, with Experimental Validation. In Int. IEEE Conference on Intelligent Vehicles Symposium (IV), pages 678-683, Gold Coast, 2013a.

Gilles Tagne, Reine Talj, and Ali Charara. Immersion and Invariance Control for Reference Trajectory Tracking of Autonomous Vehicles. In Int. IEEE Conference on Intelligent Transportation Systems (ITSC), pages 23222328, The Hague, 2013b.

\section{ACKNOWLEDGEMENTS}

We would like to thank Rogelio Lozano for our exchange about the theory of passivity, which has been a great help for us. 